\title{
Research Paper \\ Qualitative Study of Relationship With God in Old Age
}

\author{
${ }^{*}$ Abdolah Motamedi ${ }^{1}$ \\ 1. Department of Clinical Psychology, School of Psychology and Educational Sciences, Allameh Tabataba'i University, Tehran, Iran
}

\begin{tabular}{l|l}
$\begin{array}{c}\text { Use yur devic to scan } \\
\text { and read thearticle online }\end{array}$ \\
12(1):68-77. http://dx.doi.org/10.21859/sija-120166 \\
doi: $:$ http://dx.doi.org/10.21859/sija-120166
\end{tabular}

Received: 06 Sep. 2016 Accepted: 10 Jan. 2017

Key words: Relationship with God, Aging, Religion

\section{A B STRACT}

Objectives The study was performed to investigate the quality of the relationship of the elderly with God. The relationship of humans with God is an ancient one and determines human relationships with self, one another, and the universe. Those who have a positive relationship with God, have less anxiety and mental disorders in the face of difficulties.

Methods \& Materials We used Grounded Theory to answer the research questions. In this regard, an open-ended questionnaire was designed and administered by means of interview. The study sampling was theoretical and continued to reach the data saturation. The total sample consisted of 18 elderly people aged between 60 and 81 y ears.

Results The findings revealed 105 concepts, 24 subcategories, and 5 main categories. The categories were analyzed in a paradigm: causal condition; phenomena-content (context); mediator condition; strategy of action/reaction; and consequences. Finally, a model of connection of categories was presented.

Conclusion The final model suggests a wide variation in relation to the quality and quantity of relationship with God. The role of the emotional, cognitive, and social factors in the emergence of this behavior (relationship with God) was clear and in accordance with the mediator conditions and the perceived consequences of the relationship.

\section{Extended Abstract}

\section{Objectives}

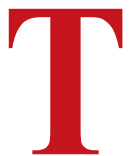

he relationship that people establish with God and the impact of this relationship on them is an essential element in the analysis of the people's character. In principle, it is known that all people communicate with God in different ways, but the quantity and quality of this relationship differ from person to person. The current study was conducted with the aim of evaluating the quality of this relationship among the elderly. It is expected that the relationship with God in old age is an indication of their affiliation to religious teachings and can be interpreted on the basis of religious concepts. Furthermore, the relationship of human being with God is very old and determines human relationships with one another, self, and the universe. People who have a positive relationship with God show less anxiety and undergo reduced psychological turmoil when faced with problems.

\section{Methods \& Materials}

To conduct this research, we considered qualitative research methods, and used the Grounded Theory. An open-ended questionnaire was designed in a semi- 
structured form and administered through interview. The interview included a combination of pre-determined, open end, and flexible questions. The sample was a theoretical sample and were selected in accordance with the study objectives. The selection continued until data saturation. In this way, a total of 18 old people, 60-81 years were selected. The obtained information was processed in three phases of open coding, key coding, and selective coding. Open coding is a manner of examining, comparing, and processing data that leads to the conceptualization and processing issues. In this step, dozens of concepts were identified and assigned into groups. Here, the categories are identified, and processed. Thus, the units that the researcher had to deal with were reduced. This was a part of key coding. Also, in the key coding phase, the categories were organized i.e., they were connected using a paradigm. In the final step, selective coding, a category is given the most importance and the remaining categories are linked to it until the theory is formulated. Here, the obtained categories in the paradigm such as causal conditions, phenomenon-content (context), mediator conditions, action/reaction strategy, and consequences were analyzed. Then, the relationships among the categories were drawn as outlined in the pattern of interaction with God (Figure 1).

\section{Results}

The obtained results revealed 105 concepts, 5 main categories, and 24 subcategories. The obtained categories and subcategories were as follows: 1) content of communication with God (including demand, praise, complaint, supplication, and self-talk); 2) importance of the relationship with God (including giving meaning to life, satisfaction of needs, personal growth, social development, and soothing); 3) causes of relationship with God (including paying attention to the consequences of a relationship with God, plight-happiness, knowing and believing in God, education, age, and time); 4) purpose of a relationship with God (including dealing with problems, immediate benefits and earning future benefits, and aimless); and 5) method of communication with God (worship, activities, exposure to situations and mediated). As mentioned earlier, the obtained categories were analyzed in a given paradigm and a model of the relationship between the categories was extracted. According to this model, three factors conditioned the relationship with God. These factors either had an accelerating or decelerating effect. Based on the findings, the factors of age, education, time, and individual experiences performed a mediating role (Figure 1).

The study showed that respondents knew different ways to communicate with God, beyond behaviors such as saying prayers, praying, etc. They also knew that performing good deeds has some kind of relationship with God. The majority of respondents focused on the role of a comforting relationship with God and believed that this relationship with God was a source of support and companionship. Achieving excellence, the feeling of being, possibility of having their heart out, and visualizing God on their side were characteristics mentioned by the elderly. The results also showed that

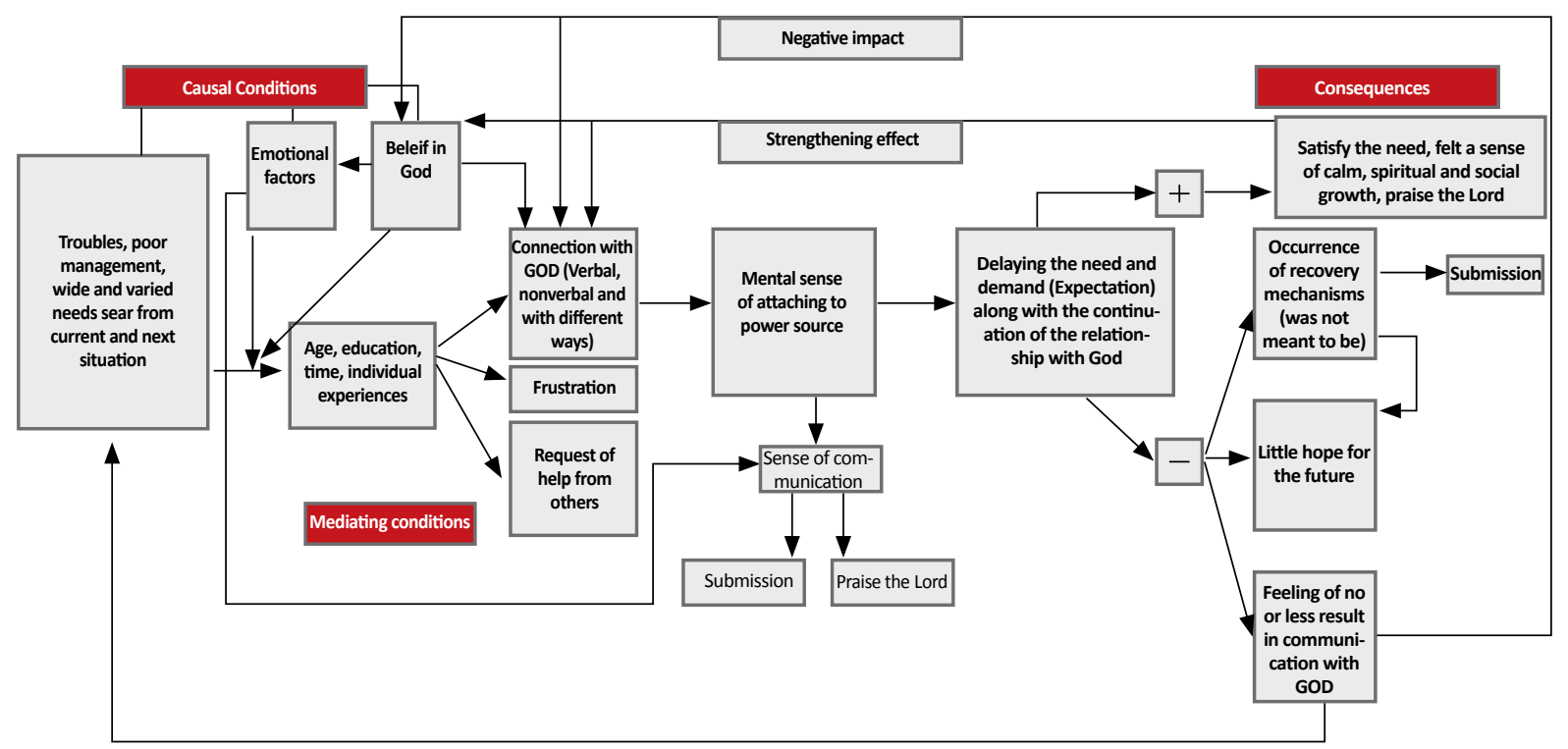

Figure 1. Interactive model of relationship with God 
with old age, needs with regard to age, privacy, faith, family, social and old conditions shape their opinions and behaviors before God. As the old people pay attention to their personal issues like asking God not to be humiliated, reaching to salvation, bestow forgiveness and so on, they pray for others, including their children and the family.

Old people believed that their knowledge of God would get better over time. Although the vast majority of them said that in the period before old age they had a relationship with God, their current relationship was more informed and based on deep understanding. Some pointed to the role of time and believed that in the past, everyone was more attached to God.

\section{Conclusion}

Using the model of causal factors, this study could provide mediated conditions, action/reaction and the implications of the relationship with God in old people. This study also presented an appropriate model for this relationship (model of interaction with God is provided below). Based on the findings of this model, it comes to light that different people have different ways to communicate with God. Moreover, under the influence of causal and consequential conditions of communicating with God, a person may also have different feelings with regard to the relationship with God. These feelings have been presented as a graphical model, keeping in mind the categories identified as a result of the paradigm introduced. In this model, three categories (1. Believe in God, 2. Emotional factors and a collection of predicaments; and 3. Needs and fears) have been introduced as factors which condition the causal relationship with God. These factors show their role in three ways in the mediating context, where they have accelerating or decelerating effect.

\section{Acknowledgments}

This research was extracted from a reasearch project funded by Allameh Tabataba'i University, Tehran, Iran.

\section{Conflict of Interest}

The authors declared no conflicts of interest. 


\section{بررسى كيفى ارتباط با خداوند در دوره سالمندى}

'عبدالله معتمدى"

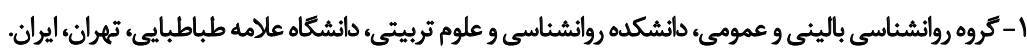

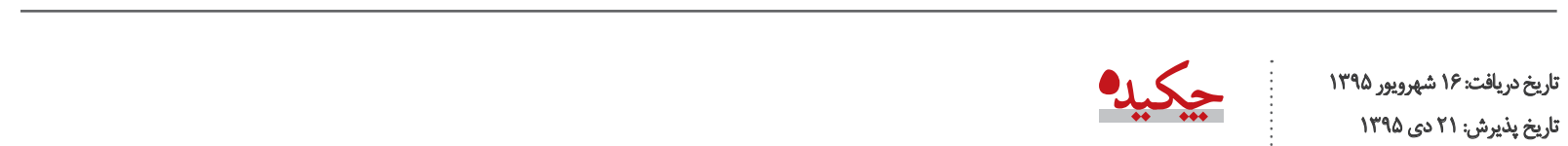

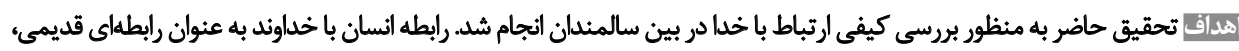

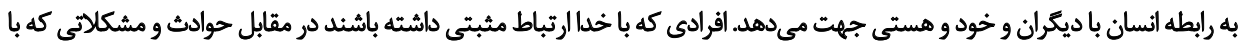

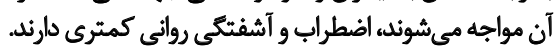

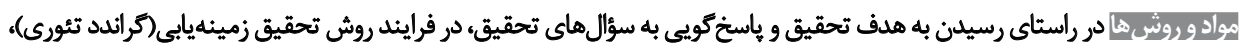

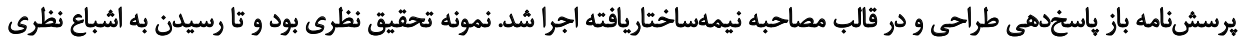

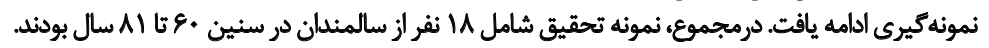

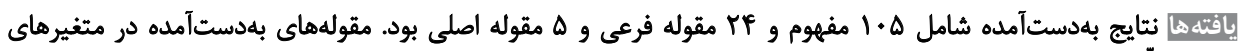

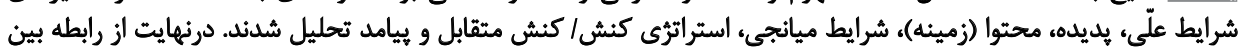

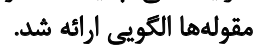

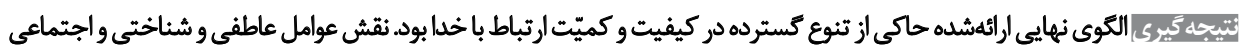

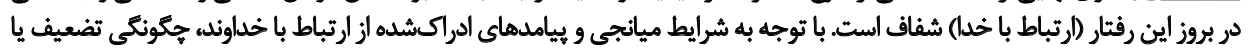
تقويت ارتباط با خداوند تروسيم شئد

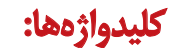

ارثباط با خداه سالمندى، مذهب

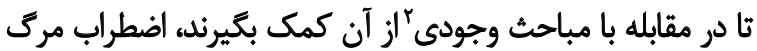

مقدمه

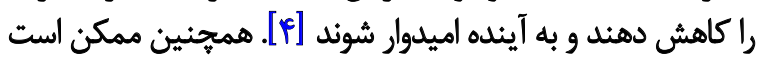

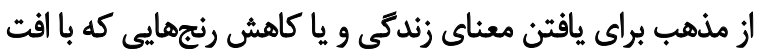

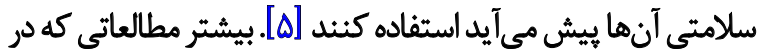

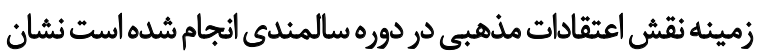

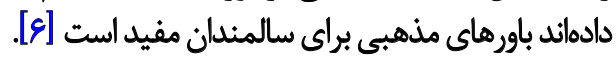

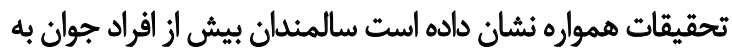

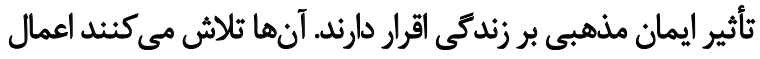

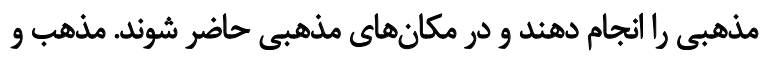

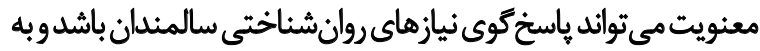

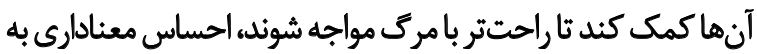

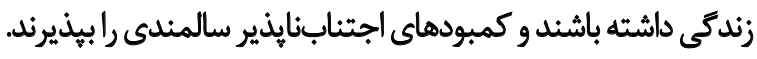

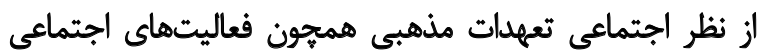

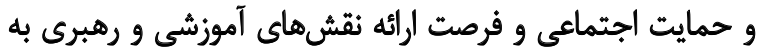

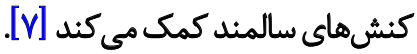

رابطهاى كه فرد با خداوند برقرار مى كند و تأثيرى كهاين رابطه روى

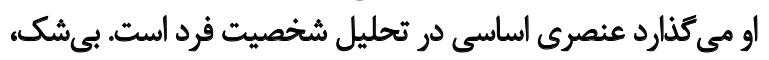

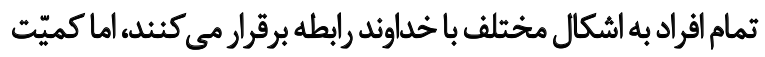

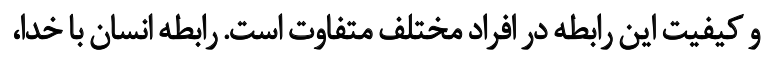

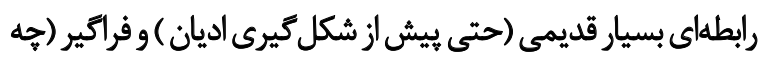

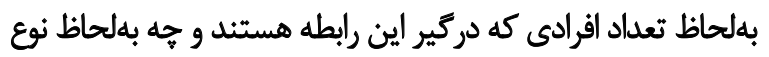

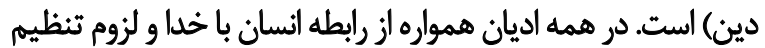

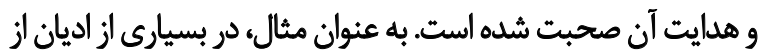

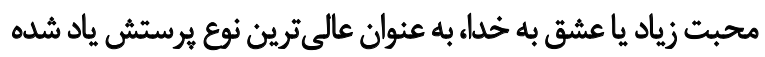

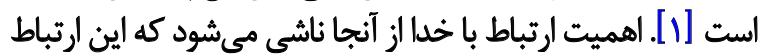

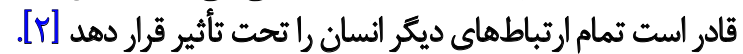

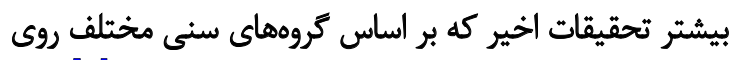

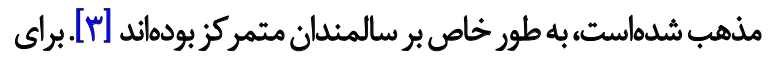

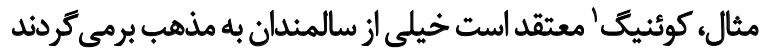

1. Koenig

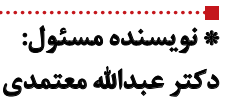

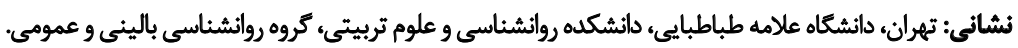

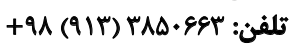

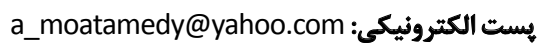


ا. رابطه وجودشناختى: رابطه هستىشناختى به اين معنى است

كه خدا تنها آفرينيده انسان است و به او هستى بخشيده است.

r. رابطه تبليغى يا ارتباطى: اين رابطه به ارتباط شفاهى و

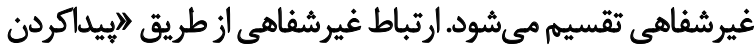

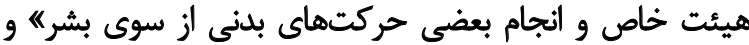

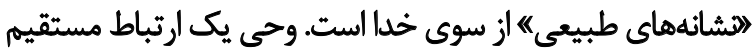

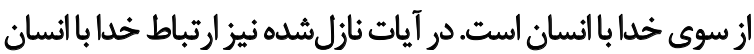

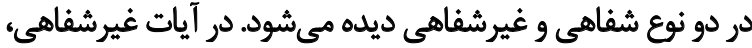

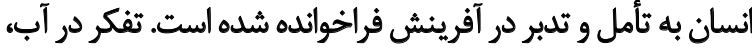

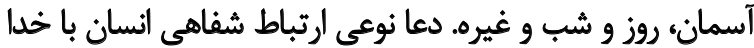

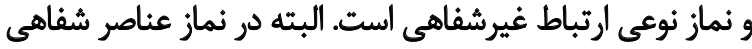

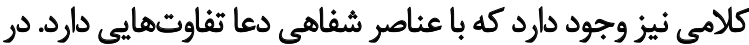

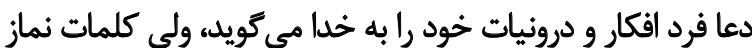
جنبه نمادين داردو قابل تغيير از سوى فرد نيات فيست

r. رابطه اخلاقي: خداوند بخشئده، مهربان و خيرخواه انسان است

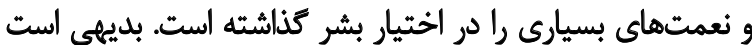

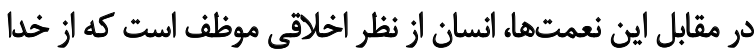

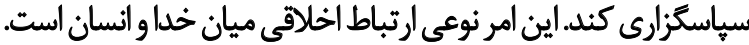

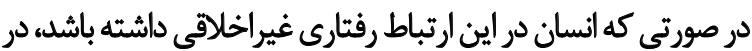

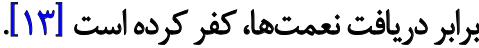

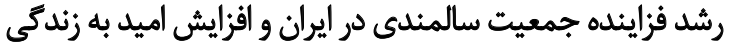

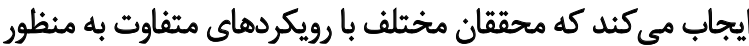

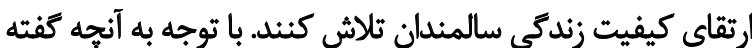

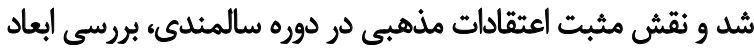

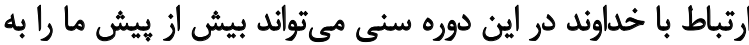

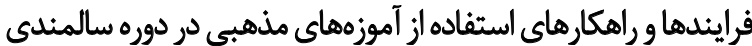

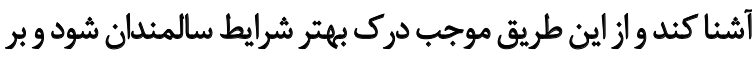

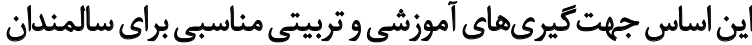
و حتى كروههاى سنى ديكر تدارك ديده شود.

انتظار اين است كه ارتباط با خدا در دوره سالمندى شاخصى از

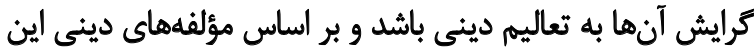

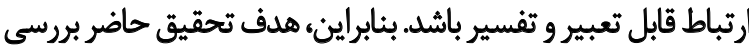

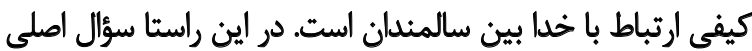

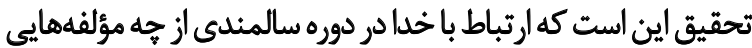

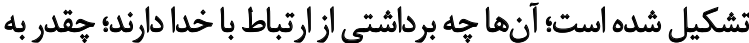

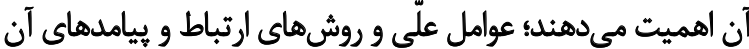

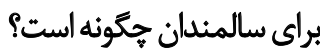

$$
\text { روش مطالعه }
$$

در راستاى رسيدن به باسخ سؤالهاي اساسى تحقيق، روش كيفى

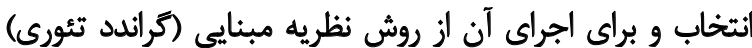

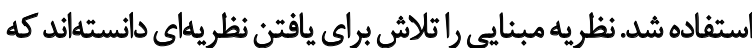

بيشتر تحقيقاتى كه در آمريكا و كشورهاي اروبايى انجام شده

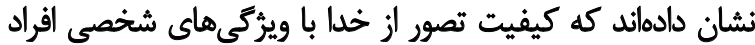

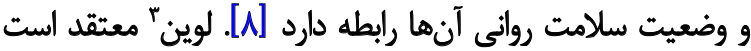

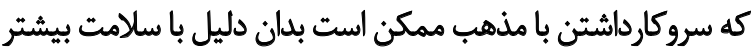

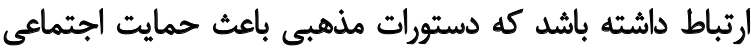

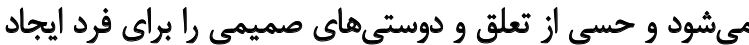

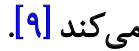

موبرك" در زمينه رابطه مذهب و سلامت شواهدى را ارائه كرد

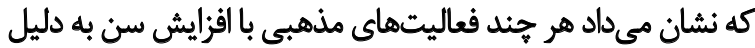

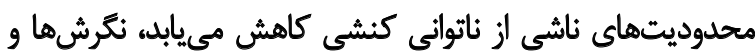

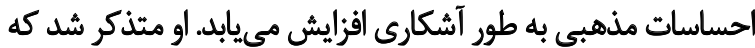

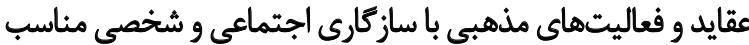

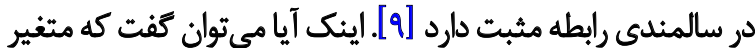

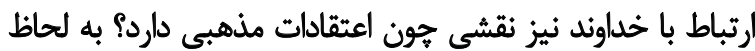

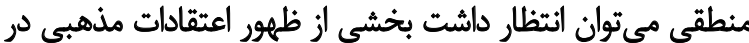

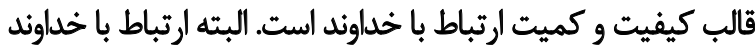

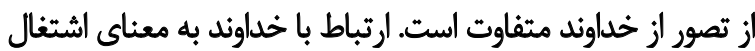

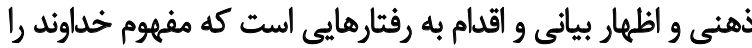

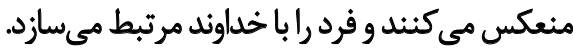
افرادى كه با خدا ارتباط مثبتى دارنده حوادث و مشكلاتى راكه

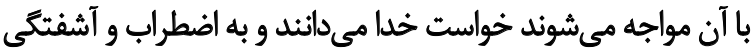

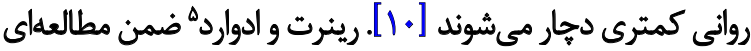

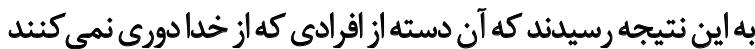

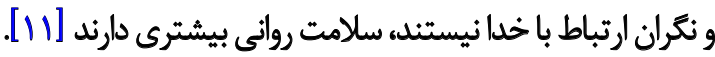

يكى از نظرياتى كه در زمينه مفهوم ارتباط با خدا در حوزه

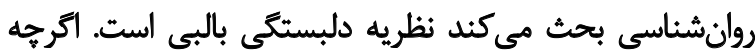

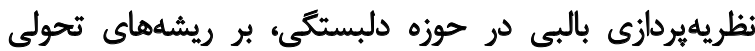

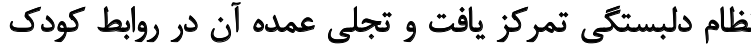

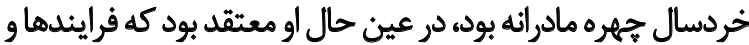

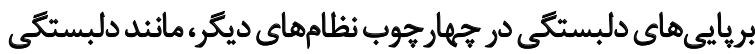

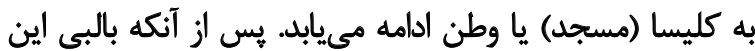

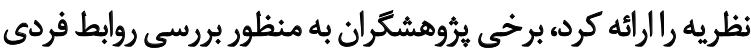

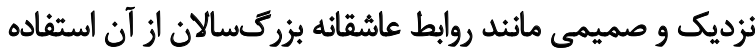

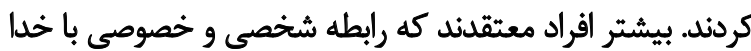

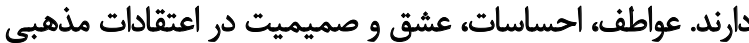

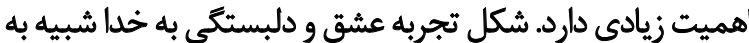

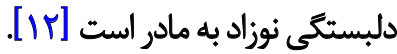
ايزوتسو (9\&F) سه نوع رابطه ميان انسان و خدا را بيان مىكند

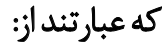

\section{Levin (1994)}

4. Moberg (1965)

5. Reinertf \& Edwards (2009) 
ابعادشان مورد توجه قرار كرفت و مدل نهايي طراحى شد.

Ladiاف

در راستاى انجام كدتذارى باز هـ • أمفهوم از مصاحبهها استخراج

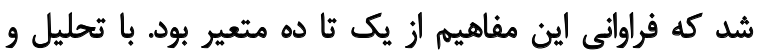

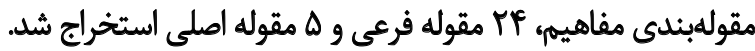

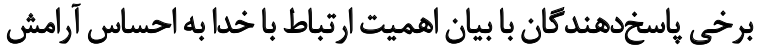

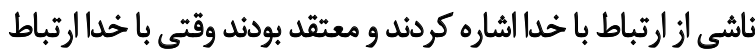

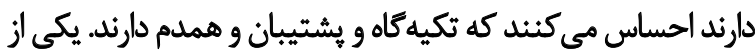

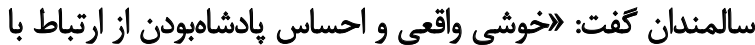

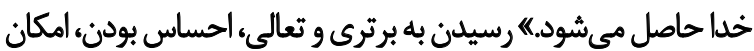

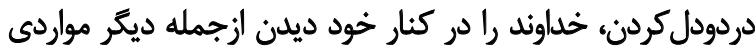

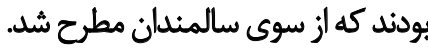

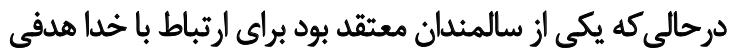

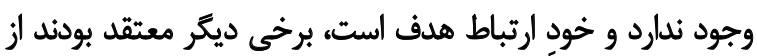

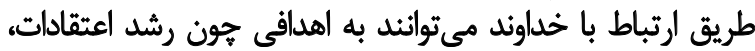

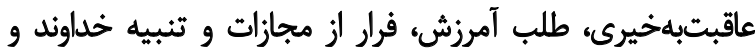

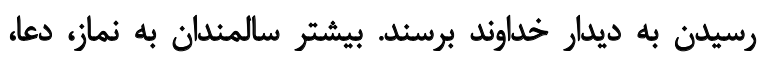

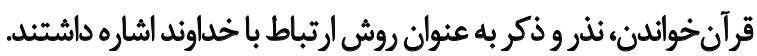

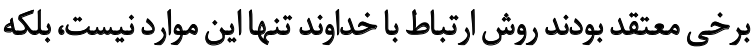

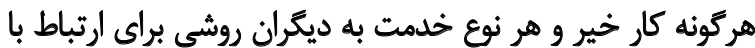

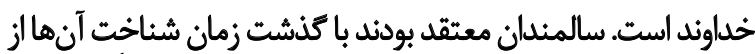

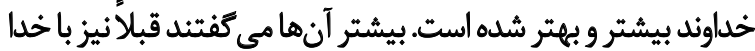

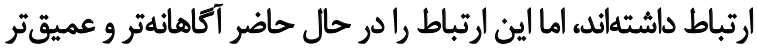

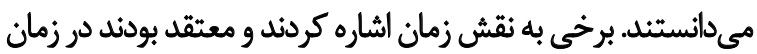
قديم نسبت به امروز مردم بيشتر به خدان ايمان داشتيند.

بخش زيادى از ارتباط با خدا در دوره سالمندى به مطالبات و

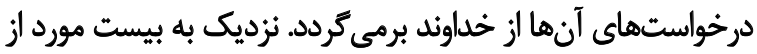

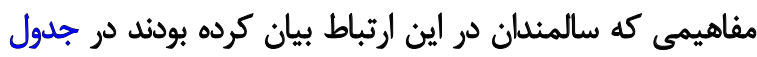

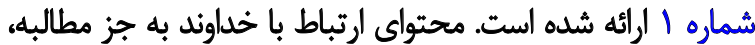

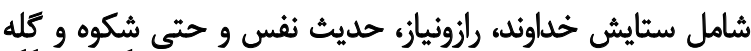

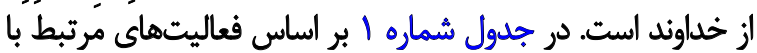

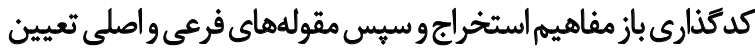

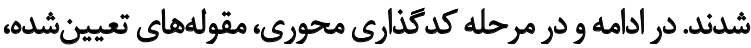

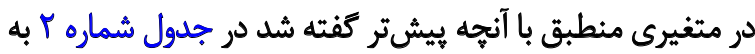
يكديكر مرتبط شدند.

در مرحله آخر يعنى مرحله كدكذارى انتخابي، يك مدل تصويرى

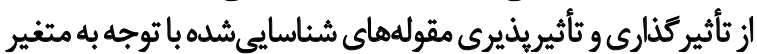

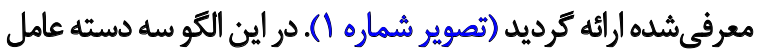

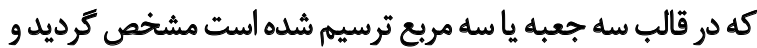

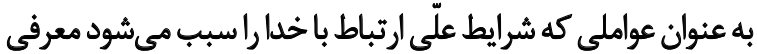

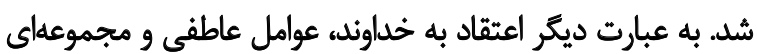
از كرفتارىها و نيازها و ترسها، برانتيزيزانده ارتباط فرامل فرد با خداوند

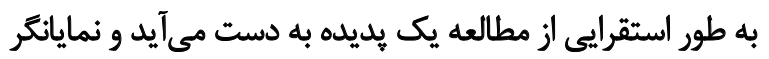

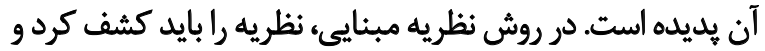

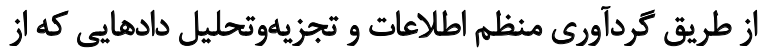

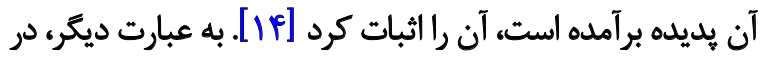

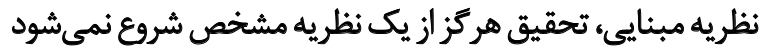

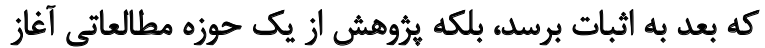

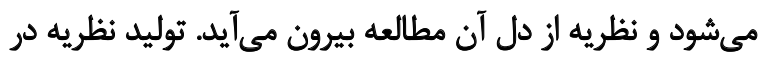

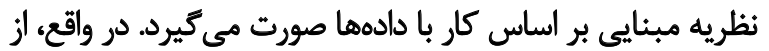

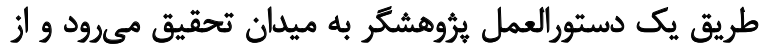
دادهها، نظريهاي را بيرون مى كشد.

در نظريه مبنايى از نمونهكيرى نظرى أستفاده مى شيود و نمونهها

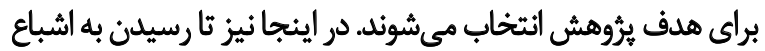

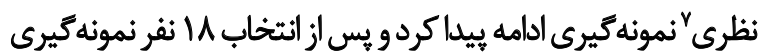

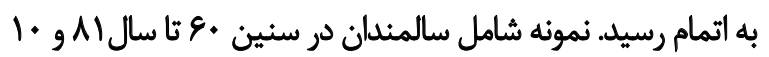

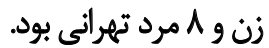

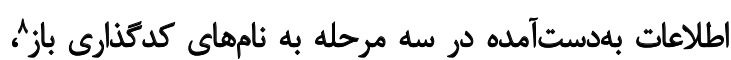

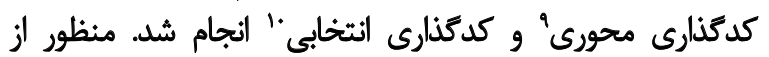

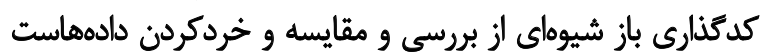

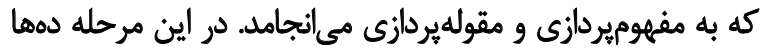

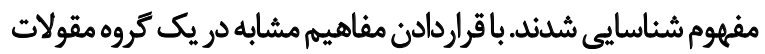

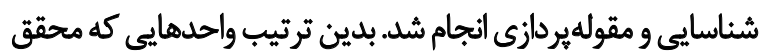

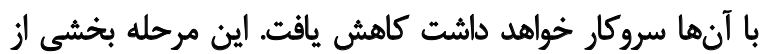

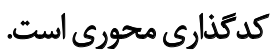

در مرحله كدكذارى محورى مقولات سازماندهى شدنده يعنى يعنى

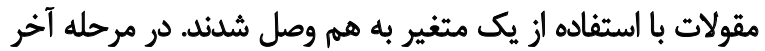

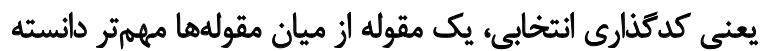

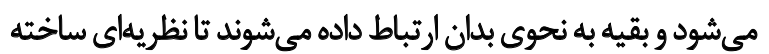

و ويرداخته شود [IF]

در اين تحقيق اطلاعات مورد نياز از طريق مصاحبه نيمهساختدار

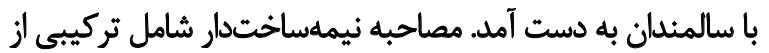

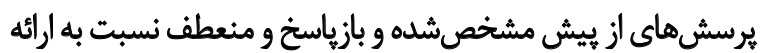

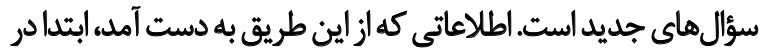

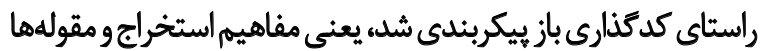

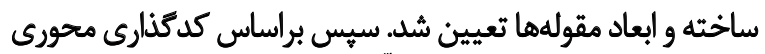

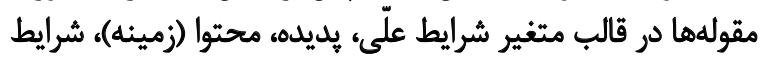

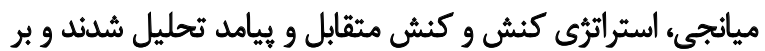

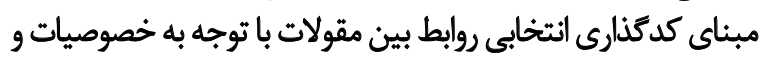

6. Theoretical sampling

7. Theory saturation

8. Open coding

9. Axial coding

10. Selective coding 
جدول ا. كدكذارى باز (استخراج مفاهيم و مقولهبندى)

\begin{tabular}{|c|c|c|}
\hline مفاهيم & مقولهاى فرعى & مقولههاى اصلى \\
\hline 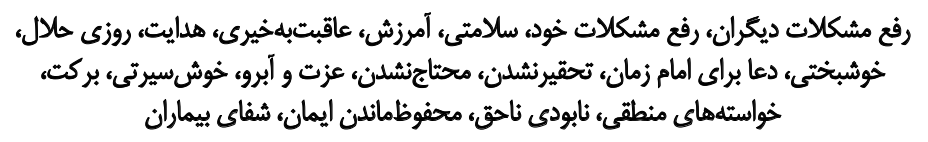 & مطالبه & \\
\hline 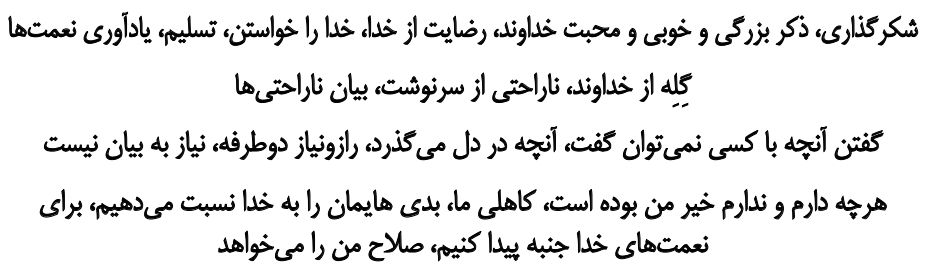 & ستايش & محتواي ارتباط با خدا \\
\hline 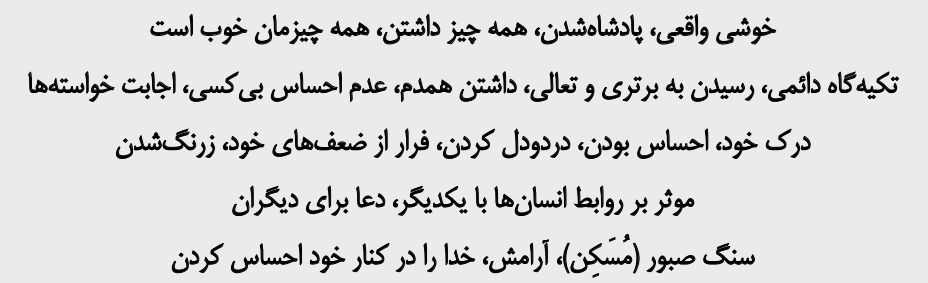 & 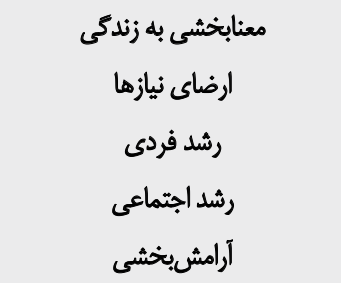 & اهميت ارتباط با خدا \\
\hline 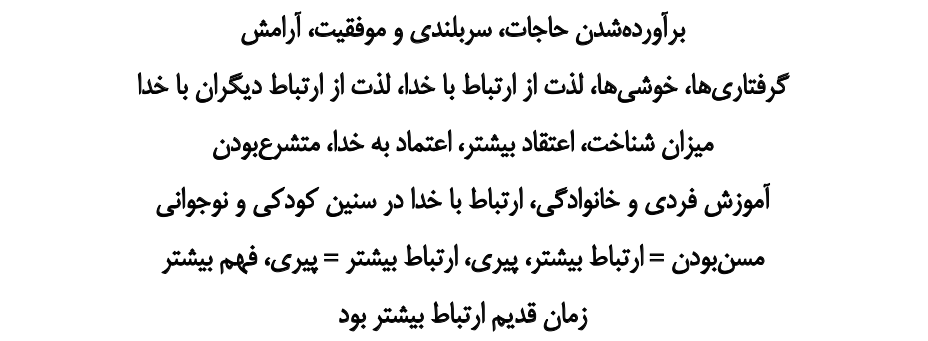 & 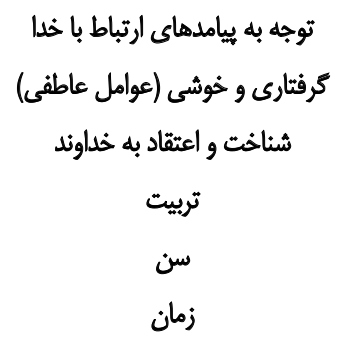 & علل ارتباط با خدا \\
\hline 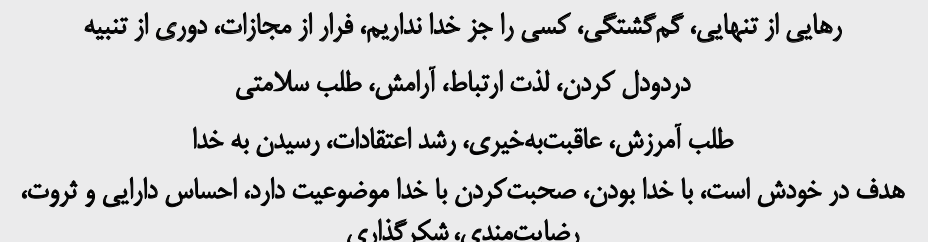 & كقابله با مشكلات كسب منافع آنى منافع آتئي & هدف ارتباط با خدا \\
\hline 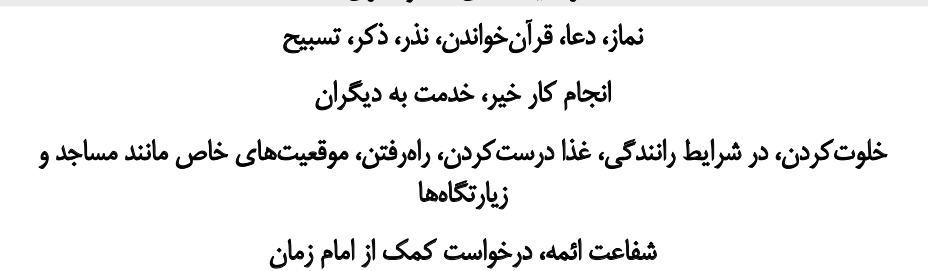 & قراركرفتن در موقعيتها & روش ارثباط با خدا \\
\hline
\end{tabular}

战

سوى خدا نرود و براى حل مشكلش از ديكَان كمك بخواهد يا آنكه از همه جا ناميد شود و احساس يأس كند، اما اكر با با خداوند إرتباط

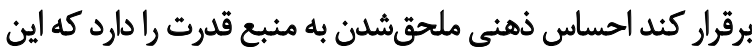

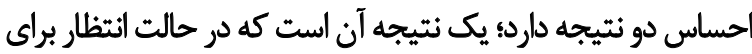

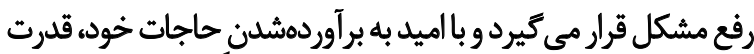

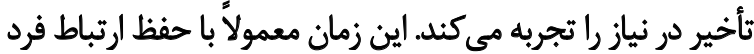

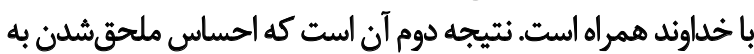

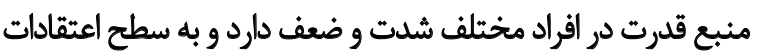

هستند. اين عوامل در بستر شرايط ميانجى يعنى شرايطى كه نقش

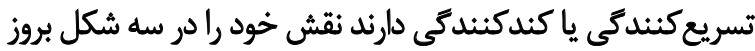

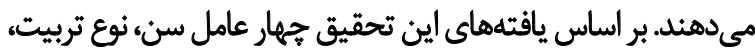
زمان و تجريههاي فرد نقش ميانجي يافي دارند. به عنوان مثال، فردى كه با مشكل و كرفتارى مواجه مي شود، براي

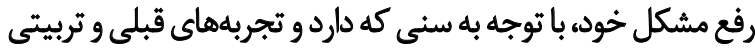

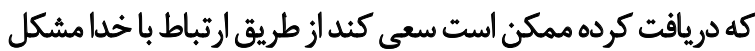

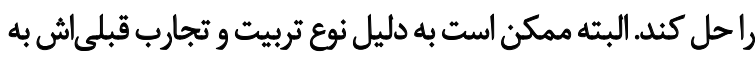


جدول ז. ارتباط با خدا، سازماندهي مقولات در مثغير روش نظريه مبنايي

\begin{tabular}{|c|c|c|c|c|c|}
\hline & ترس ها & نيازها & 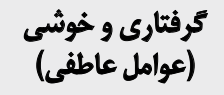 & شئ خاخت و اعثتاد & عوامل مؤثر بر ارتباط با خميلى \\
\hline & & 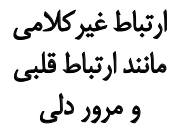 & و صحبت كردن با خلاون هائد & ارثباط با خداوئد & يليله \\
\hline \multirow[t]{3}{*}{ ستايش } & شِكوِه & حديث نقس & الزوونياز & مطالبهك كرى & أثكيزه ارتباط برقراركنندكان با خدا در بين سالمندان \\
\hline & & ارتباط با واسططه & فعاليتهاي ديكر & عبادت & با هدف مقابله با مشكلات و وكسب منافع آنى و آتي \\
\hline & 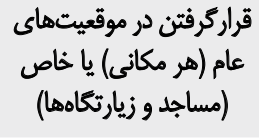 & ترييت & زمان & ارتباط با واسطه & عوامل مؤثر در تسريع يا عدم تسريع ارتباط با خلا \\
\hline آرامش بخششى & رشد اجتماعيى & رشد فردى & ارضاي نيازها & زعنابخشى بله & ييامدهاي ارتباط با حُدا \\
\hline
\end{tabular}

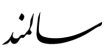

رفتتارهايى جون نمازخواندن، دعاكردن و امثال اين موارد ميدانستند

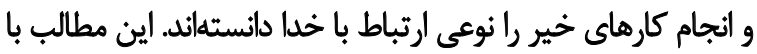

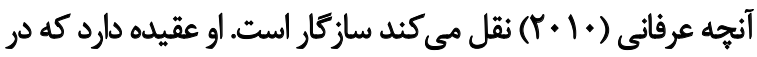

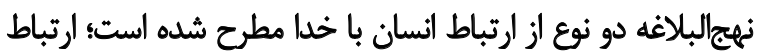

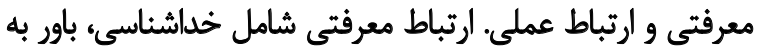

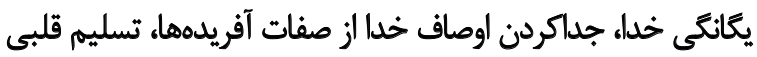

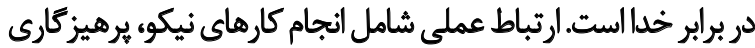

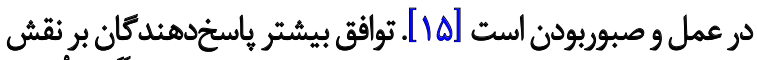

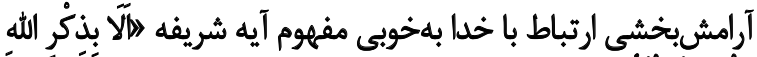

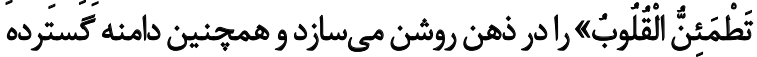

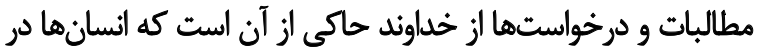

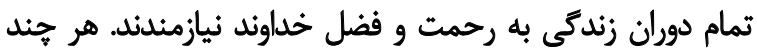

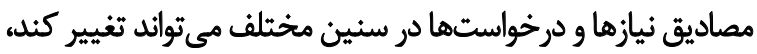

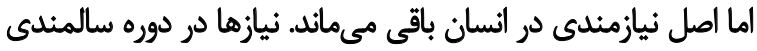

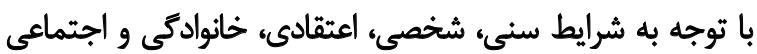

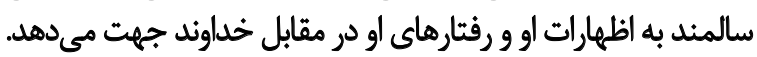

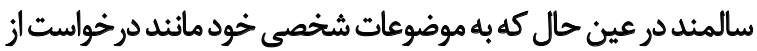

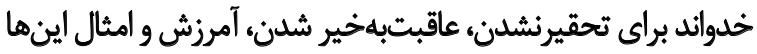

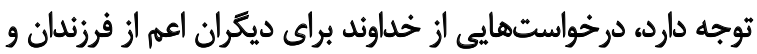

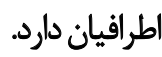

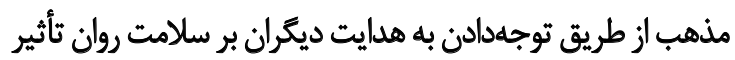

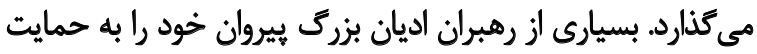

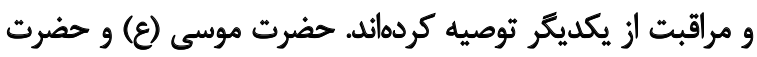

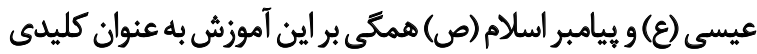

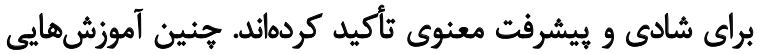

آنها بستكى داردء احساس بهرهبردن درونى ناشى از ارتباط است. اين احساس، ستايش خداوند و تسليم را در فردد زينده مي كئد.

همانطور كه كَفته شد، بر اساس نتيجه اول فرد در حالت انتظار

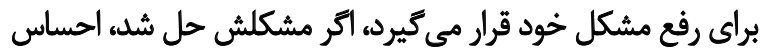

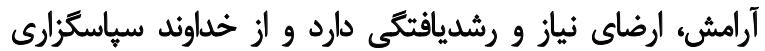

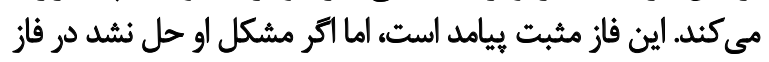

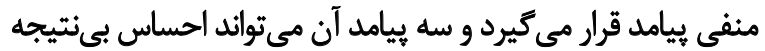

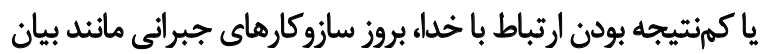

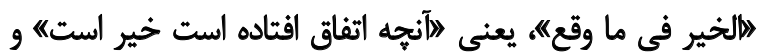

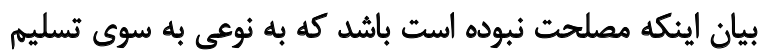

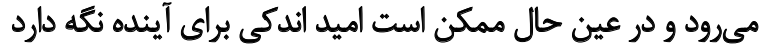

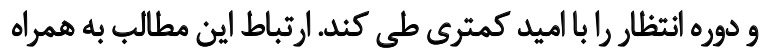

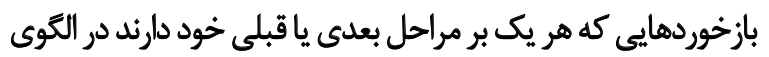
تعاملى ارتباطى مشخص شده است.

$$
\text { بحث }
$$

هدف تحقيق حاضر بررسى موضوع ارتباط با خدا در بين سالمثدان

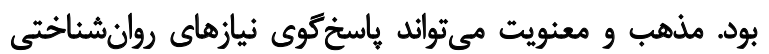

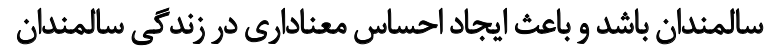

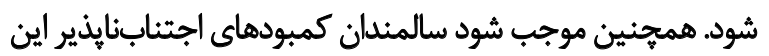

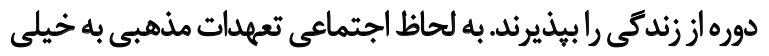

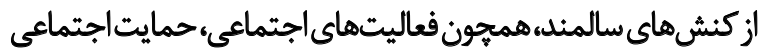

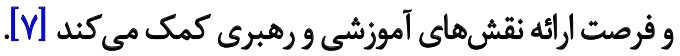
در اين تحقيق باسخدهندكان شيوه ارتباط با خدا را فراتر از 


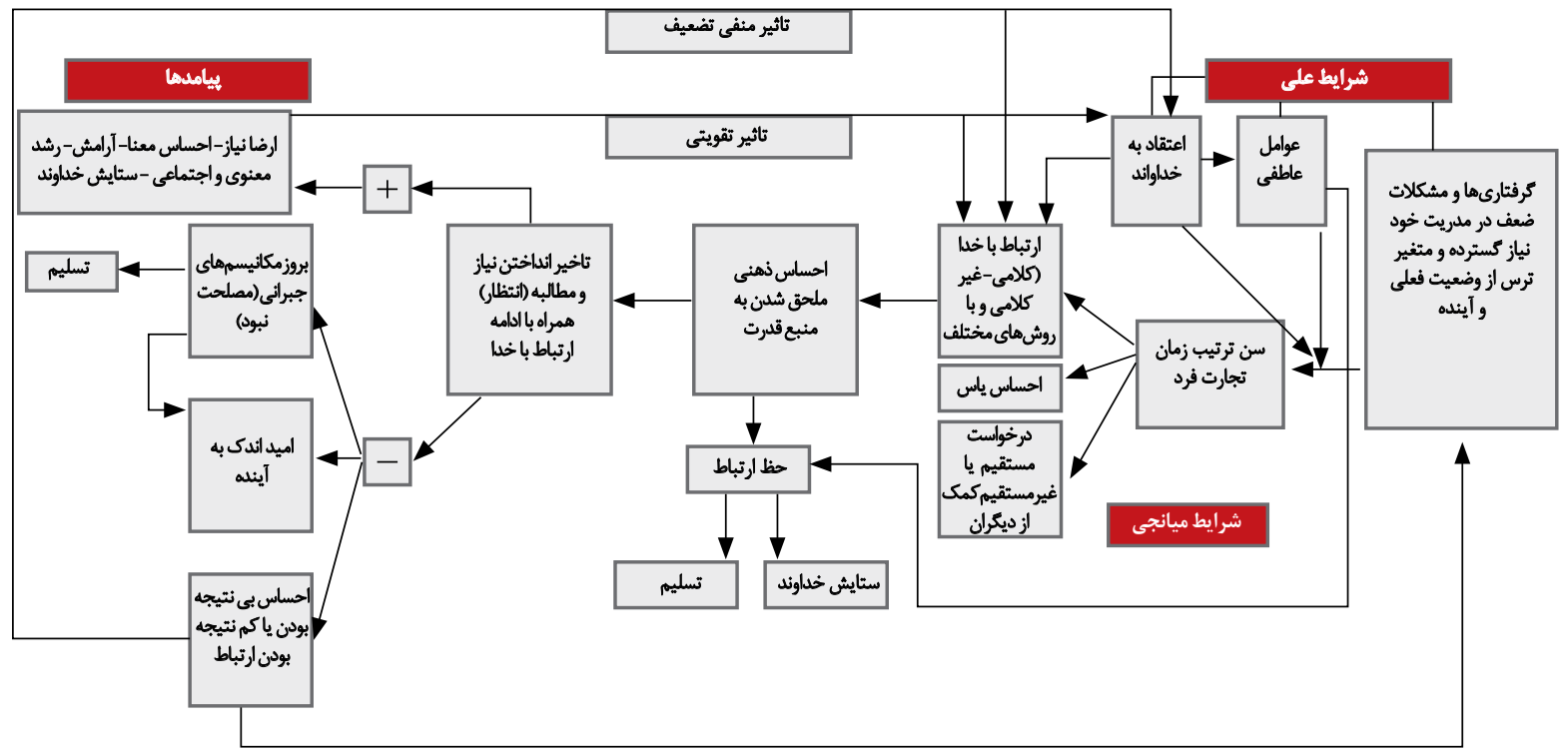

مائد سن و تربيت و تجربههاى فرد كه مي توانئد عامل تسهيل كنئده

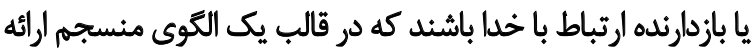

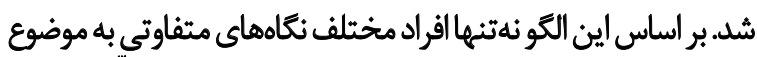

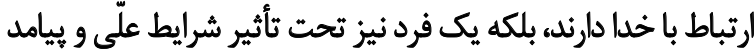

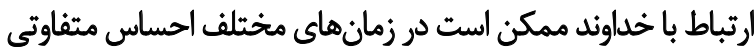

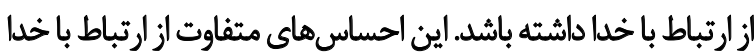

$$
\text { در قالب اين مدل قابل توضيح است. }
$$

مههمترين محدوديت تحقيق حاضر مبتنى بر متدولوزيى تحقيق به

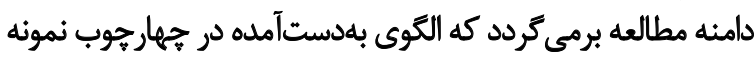

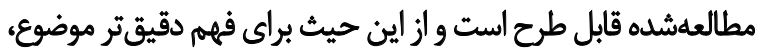

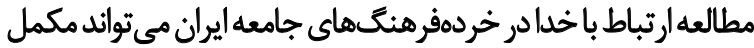

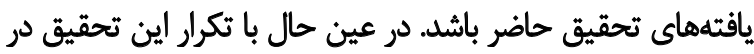

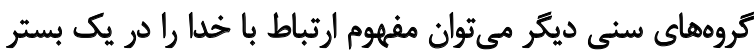

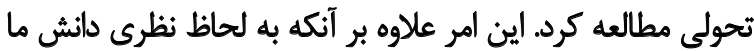

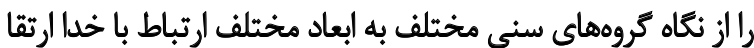

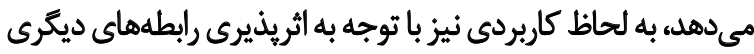

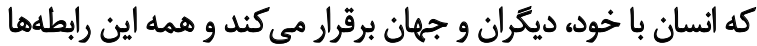

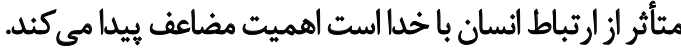

$$
\text { تشكر و قدرواني }
$$

مقاله حاضر از يك طرح بثوهشى ترفته شده است كه با تصويب و

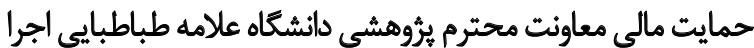

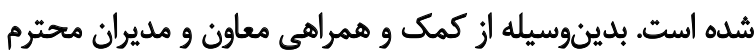

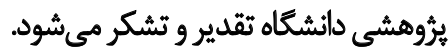

بخشش، رحمت، مهربانى، غمخوارى و سخاوت را در مقابل ديكران

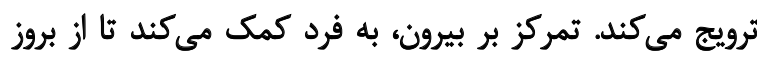

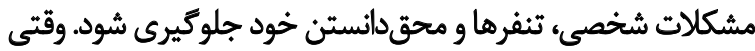

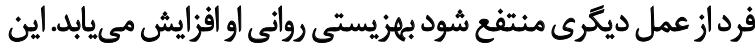

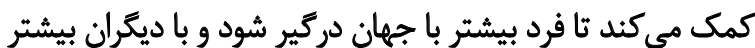

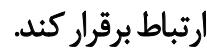

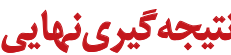

در راستاى رسيدن به هدف تحقيق و ياسخ به اين سؤال اساسى كه

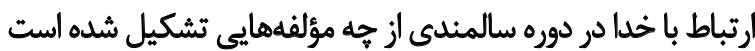

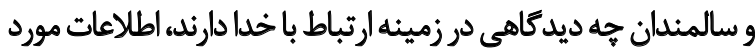

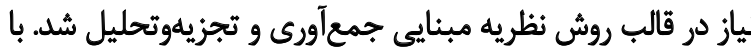

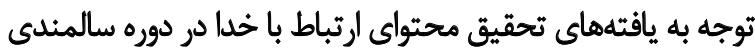

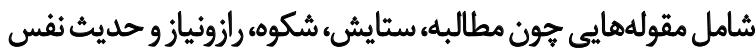

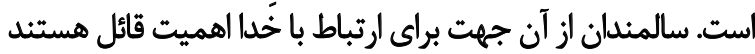

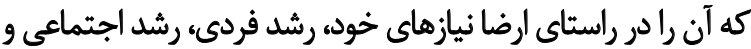

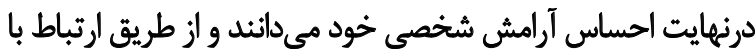

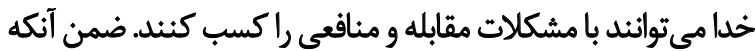

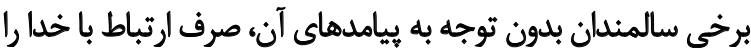

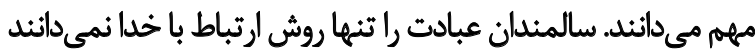

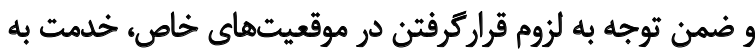

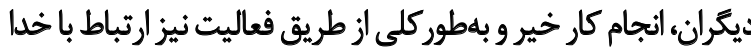

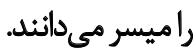

اين تحقيق با مدل مناسب، عوامل علّى، شرايط ميانجى، كنش و و روني

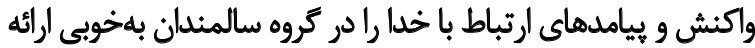

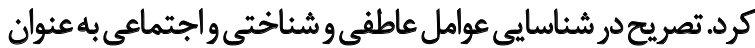

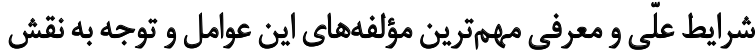

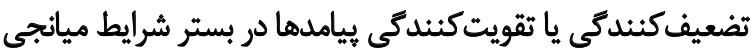




\section{References}

[1] Mazaheri, MA, Pasandideh A, Sadeghi MS. [Faith triangle (Dimensions of man's relationship with God) (Persian)]. Qom: Darolhadis Publishers; 2010

[2] Nasri A. [Anthropological principles in the Quran (Persian)]. Tehran: Publishing Organization Institute for Islamic Culture and Thought; 2005.

[3] Krause N. Stressors in highly valued roles, religious coping, and mortality. Psychology and Aging. 1998; 13(2):242-55. doi: 10.1037/0882-7974.13.2.242

[4] Koenig HG, Weiner DK, Peterson BL, Meador KG, Keefe FJ. Religious coping in the nursing home: A biopsychosocial model. The International Journal of Psychiatry in Medicine. 1997; 27(4):365-76. doi: 10.2190/m2d6-5ydg-m1dd-a958

[5] Musick MA, Blazer DG, Hays JC. Religious activity, alcohol use, and depression in a sample of elderly baptists. Research on Aging. 2000; 22(2):91-116. doi: 10.1177/0164027500222001

[6] Lloyd P. The psychology of ageing: An introduction. British Journal of Social Work. 2007; 37(3):584-6. doi: 10.1093/bjsw/ bcm037

[7] Santrock JW. Life span development. New York: McGraw-Hill; 2013.

[8] Bonab BG, Koohsar AAH. Relation between quality of image of God with hostility and interpersonal sensitivity in parents of exceptional children. Procedia - Social and Behavioral Sciences. 2011; 29:242-6. doi: 10.1016/j.sbspro.2011.11.232

[9] Levin J. Religion in aging and health: Theoretical foundations and methodological frontiers. California: SAGE Publications, Inc; 1994. doi: $10.4135 / 9781483326641$

[10] Laurin K, Kay AC, Moscovitch DA. On the belief in God: Towards an understanding of the emotional substrates of compensatory control. Journal of Experimental Social Psychology. 2008; 44(6):1559-62. doi: 10.1016/j.jesp.2008.07.007

[11] Reinert DF, Edwards CE. Attachment theory, childhood mistreatment, and religiosity. Psychology of Religion and Spirituality. 2009; 1(1):25-34. doi: 10.1037/a0014894

[12] Krikpatrick LA. Attachment and religious representation and behavior. In: Cassidy J, Shaver PR, editors. Handbook of Attachment. New York: Gilford Press.

[13] Izutsu T. God and man in the Quran. Petaling Jaya: Islamic Book Trust; 2002.

[14] Corbin J, Strauss A. Basics of qualitative research: Techniques and procedures for developing grounded theory. California: SAGE Publications, Inc; 2008. doi: 10.4135/9781452230153

[15] Erfani GhH. Ways to communicate with God from the perspective of Nahjolbalaghe. Afagh-e Mehr. 2010; 9(48):24-26. 